\title{
ATUALIZAÇÃO EM GIST
}

\author{
UPDATE IN GIST
}

Eduardo Linhares, TCBC-RJ ${ }^{1}$; Marcus Valadão, TCBC-RJ ${ }^{2}$

\section{INTRODUÇÃO}

Até há 20 anos atrás acreditava-se que a maioria dos tumores mesenquimais gastrointestinais eram provenientes da musculatura lisa, ${ }^{1}$ sendo denominados de "leiomiomas" e "leiomiossarcomas". A utilização da microscopia eletrônica e da imunohistoquímica, entretanto, evidenciou que apenas alguns desses tumores apresentavam características de diferenciação de músculo liso, contribuindo para adoção do termo mais genérico, tumor estromal, proposto por Mazur e Clark ${ }^{2}$ em 1983. Posteriormente, alguns autores demonstraram que esses tumores também apresentavam características de diferenciação neuronal, ${ }^{3,4}$ designando-os de "plexosarcomas" e "tumor gastrointestinal do nervo autonômico".

Só recentemente foi esclarecido que essa neoplasia constitui uma entidade bem definida designada GIST (sigla em inglês para gastrointestinal stromal tumor) através das descobertas de sua origem a partir das células intersticiais de $\mathrm{Cajal}^{5}$ e da expressão da proteína c-Kit ${ }^{6}$.

As células intersticiais de Cajal são responsáveis pela motilidade intestinal, ${ }^{7}$ apresentam características imunofenotípicas e ultra-estruturais tanto de músculo liso quanto de diferenciação neural e expressam o receptor Kit (CD117) semelhante ao tumor estromal gastrointestinal (GIST). O Kit é um receptor tirosina quinase transmembrana, responsável por várias funções celulares, dentre as quais proliferação, adesão, apoptose e diferenciação celular ${ }^{8}$. No GIST, a mutação no gene Kit é responsável pela ativação constitutiva na proteína Kit, que causa estímulo sem oposição para proliferação celular, estando implicada na sua gênese.

\section{EPIDEMIOLOGIA}

Embora relativamente raro, o GIST representa $80 \%$ dos tumores mesenquimais do trato digestivo e constitui $5 \%$ de todos os sarcomas ${ }^{9}$. Ocorrem, predominantemente, em indivíduos de meia idade (média em torno de 60 anos), sendo infreqüente a ocorrência em idades extremas. Podem ter origem em todo trato gastrointestinal, sendo mais comuns no estômago $(70 \%)$, seguido pelo intestino delgado $(20 \%$ a $30 \%)$, intestino grosso $(10 \%)$ e outros locais da cavidade abdominal (5\%).

\section{DIAGNÓSTICO}

O consenso atual é que o diagnóstico de GIST é feito quando existirem quadro clínico, características morfológicas celulares típicas e imunohistoquímica positiva para c-Kit (CD117). Porém, alguns tumores (em torno de 4\%) apresentam características clínicas e patológicas compatíveis com GIST mas não expressam a proteína Kit. Heinrich et al. ${ }^{10}$ demonstraram que este grupo (GIST c-Kit negativo) apresenta mutação em outro receptor tirosina quinase com atividades semelhantes ao Kit (Receptor do Fator de Crescimento Ativado Plaquetário-PDGFRá), representando uma via alternativa na patogênese desta neoplasia.

\section{PATOLOGIA}

A predição do comportamento biológico do GIST é problemática, pois apesar da identificação na literatura de inúmeras variáveis capazes de predizer sua evolução, ${ }^{11-14}$ os achados são conflitantes, não havendo consenso. Diante disto, tem-se evitado o termo "benigno" e classificado o GIST de acordo com o potencial de malignidade com base nos dois fatores prognósticos mais relevantes reconhecidos na literatura (Tabela 1). ${ }^{15}$

Tabela 1 - Estimativa do potencial de malignidade (Fletcher e colaboradores $^{15}$ ).

\begin{tabular}{lcc}
\hline $\begin{array}{l}\text { Risco de } \\
\text { malignidade }\end{array}$ & Tamanho & índice mitótico (50cga) \\
\hline muito baixo & $<2$ & $<5$ \\
baixo & $2-5$ & $<5$ \\
intermediário & $<5$ & $6-10$ \\
& $5-10$ & $<5$ \\
alto & $>5$ & $>5$ \\
& $>10$ & qualquer índice \\
& qualquer tamanho & $>10$ \\
\hline
\end{tabular}

\section{TRATAMENTO}

A ressecção cirúrgica completa é o tratamento padrão para o GIST, pois é a única modalidade capaz de propor-

1. Cirurgião da Seção de Cirurgia Abdômino-Pélvica-INCA; Mestre e Doutor em Cirurgia Abdominal pela UFRJ.

2. Cirurgião oncológico do INCA.

Recebido em 07/11/2005

Aceito para publicação em 16/01/2006

Conflito de interesses: nenhum

Fonte de financiamento: nenhuma

Trabalho realizado no Instituto Nacional do Câncer - INCA-RJ. 
cionar cura. A ressecção R0 (ausência de doença residual) representa uma das influências mais importantes para o resultado do tratamento (intervalo livre de doença e sobrevida), sendo alcançada em torno de $40 \%$ a $60 \%$ de todos os casos de GIST e em mais de $70 \%$ nos casos de doença não metastática ${ }^{9,16}$. O objetivo primordial da cirurgia é a ressecção completa do tumor e o tipo de ressecção a ser empregada está na dependência da localização e do tamanho do tumor. As lesões com suspeita de invasão de órgãos adjacentes devem ser tratadas por cirurgia radical através da ressecção em monobloco do órgão acometido. É mandatório que a ressecção obtenha margens negativas checadas por exame de congelação intra-operatória, pois a presença de doença residual influencia negativamente a sobrevida ${ }^{17}$. Não está estabelecido qual a extensão ideal da margem cirúrgica, porém há consenso de que não é necessária margem ampla para ressecção completa da lesão. Desta forma, a depender da localização e do tamanho tumoral, a ressecção segmentar do órgão de origem da lesão pode ser empregada (geralmente factível no tratamento das lesões de origem gástrica) desde que seja respeitada a premissa da margem negativa (Figuras 1,2 e 3). É necessária uma técnica cirúrgica meticulosa visando a prevenir a rotura tumoral durante o ato cirúrgico, pois a cápsula do tumor se rompe com facilidade, podendo resultar em disseminação neoplásica e pior prognóstico ${ }^{18}$. A metástase nodal é um evento infreqüente, ${ }^{9}$ não havendo subsídio na literatura que corrobore a realização de linfadenectomia de rotina salvo na presença de linfonodos macroscópicamente suspeitos .

A ressecção laparoscópica tem sido empregada no tratamento do GIST, sendo descritas pequenas séries de casos no manejo de lesões menores que $3 \mathrm{~cm}$, conferindo vantagens como mínima manipulação tumoral e eficácia no diagnóstico e tratamento das lesões incidentais e naquelas que se apresentam com hemorragia digestiva ${ }^{19,20}$. Apesar da descrição de sucesso oncológico com ressecções laparoscópicas, ${ }^{21}$ há necessidade de estudos com maior número de casos e acompanhamento a longo prazo para se definir o papel da laparoscopia no tratamento desta neoplasia.

A presença de recidiva é freqüente apesar da ressecção cirúrgica completa do tumor primário. Na experiência do MD Anderson Cancer Center, somente 10\% dos pacientes estavam livres de doença após 10 anos de acompanhamento $^{18}$. O padrão inicial de recidiva envolve, predominantemente, o peritôneo e o fígado. Na casuística do Memorial SloanKattering Cancer Center, quarenta por cento dos pacientes submetidos à cirurgia potencialmente curativa (R0) desenvolveram recidiva, com acometimento do peritôneo em $50 \%$ dos casos e do fígado em $75 \%$, com sobrevida média de 15 meses após resgate cirúrgico ${ }^{22}$.

O resgate cirúrgico deve ser empregado sempre que seja constatada doença ressecável, porém todos esses pacientes irão desenvolver recidivas subseqüentes apesar da ressecção macroscópica completa dessas lesões. A recidiva peritoneal, geralmente, permite a ressecção completa do tumor, porém a recorrência hepática usualmente se apresenta como doença difusa intra-hepática, impedindo a abordagem cirúrgica.

O tratamento cirúrgico da doença metastática está restrito a número limitado de casos, visto que a grande maioria dos

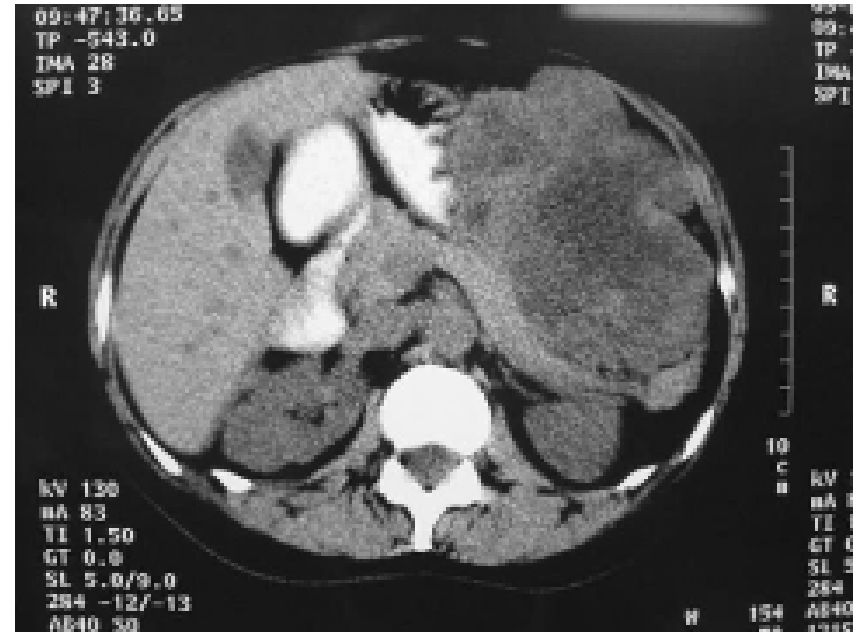

Figura 1 - Tomografia evidenciando volumoso GIST de origem gástrica.

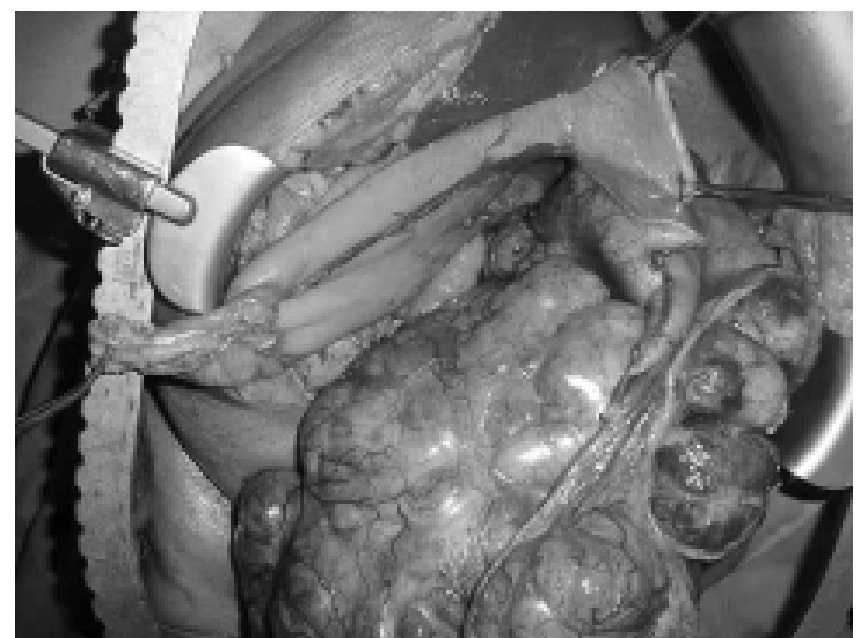

Figura 2 - Lesão proveniente da grande curvatura gástrica com invasão de cólon transverso e baço, sendo possível ressecção gástrica segmentar com margens adequadas associado a colectomia e esplenectomia em bloco.

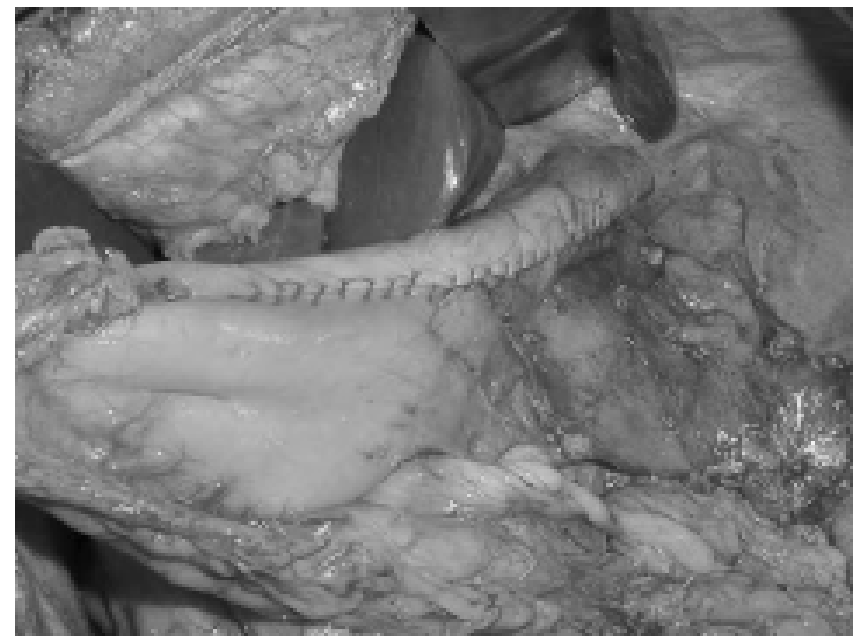

Figura 3 - Aspecto final demonstrando preservação gástrica.

pacientes com doença metastática apresenta disseminação extensa, impossibilitando a ressecção tumoral. Diante deste fato, alguns tipos de tratamento foram utilizados para controlar doença metastática irressecável como radioterapia, quimioterapia 
sistêmica ou intra-peritoneal e a quimioembolização da artéria hepática, porém sem evidência de benefício ${ }^{23}$.

A descoberta do STI571 (mesilato de imatinib [Glivec ${ }^{\circledR}$ ];

Novartis, Basel, Switzerland) revolucionou o tratamento do câncer por ser a primeira droga a agir especificamente na alteração molecular responsável pela etiologia destes tumores. O conhecimento de mutações (com ganho de função do gene Kit) na gênese e progressão do GIST proporcionou o desenvolvimento de uma droga com alvo molecular definido que interfere na atividade tirosina quinase dos receptores Kit. Inicialmente utilizada no tratamento da leucemia mielóide crônica com grande sucesso, o STI571 começou a ser utilizado no tratamento dos GIST.

O primeiro relato de tratamento do GIST com STI571 foi em fevereiro de 2000 na Finlândia ${ }^{24}$. A paciente apresentava doença disseminada na cavidade abdominal, e insucesso com várias outras modalidades terapêuticas. O tumor expressava a proteína Kit (imunohistoquímica positiva para CD117) e continha mutação no exon 11 do gene Kit. Em algumas semanas de tratamento, houve resposta dramática mensurada por tomografia (Figuras 4 e 5), PET-SCAN e biópsias seriadas do tumor. Histologicamente, o tumor foi substituído por degeneração mixóide e fibrose.

Os resultados encorajadores com o primeiro caso levaram à implementação de estudos fase I e II, ${ }^{25,26}$ evidenciando que o mesilato de imatinib apresentava atividade significante nos doentes com GIST avançado, alcançando taxa de resposta parcial em $53,7 \%$, estabilização da doença em $27,9 \%$ e toxicidade grau 3 e 4 (hemorragia, dor abdominal e distúrbio eletrolítico) em $21,1 \%$ dos casos.

$\mathrm{O}$ acompanhamento a longo prazo de portadores de GIST de alto risco submetidos à ressecção cirúrgica indica que a cirurgia isolada geralmente não é capaz de proporcionar cura, pois $85 \%$ a $90 \%$ desses casos irão desenvolver algum evento adverso (recidiva, metástase ou morte).${ }^{16}$ Em geral, mais de $50 \%$ dos pacientes submetidos à cirurgia potencialmente curativa irão desenvolver recidiva ou doença metastática ${ }^{16,27}$ e irão cursar com sobrevida reduzida.

Se por um lado está claro que o imatinib traz benefícios aos pacientes com doença irressecável ou metastática, por outro, vem à tona o questionamento se o imatinib seria bené-

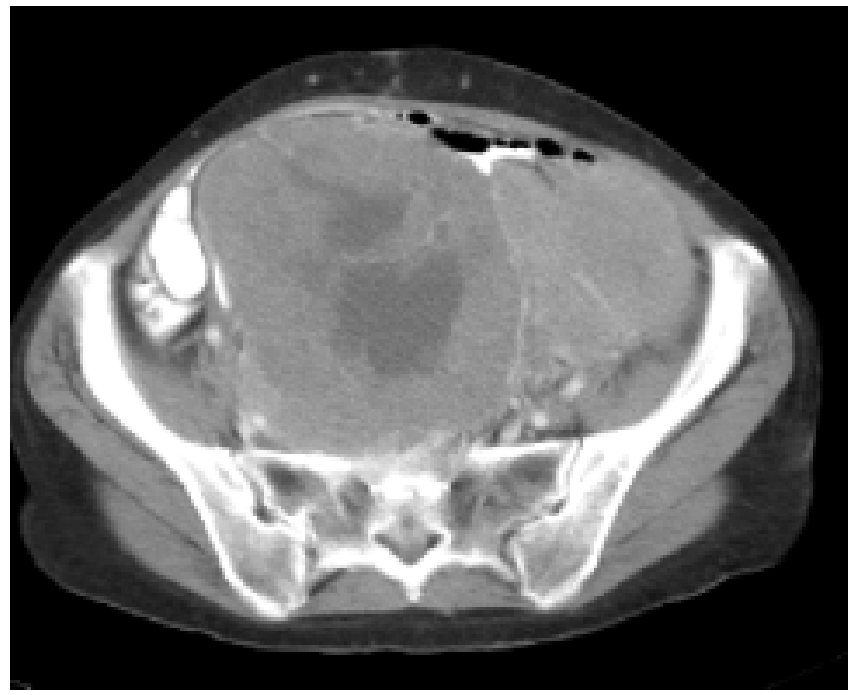

Figura 4 - Antes do imatinib. fico no subgrupo de pacientes operados que tivessem prognóstico ruim. Essa hipótese despertou o início da utilização do imatinib como terapia adjuvante e neoadjuvante e instrumento capaz de otimizar os resultados obtidos com a cirurgia.

O racional é que a terapia adjuvante/neoadjuvante com imatinib seja capaz de reduzir as chances de recidiva local e de metástase à distância, prolongar o intervalo livre de doença e a sobrevida global, aumentar o número de casos irressecáveis elegíveis para ressecção (redução tumoral farmacológica) e otimizar a resposta ao imatinib após citorredução cirúrgica.

Algumas linhas de pesquisa estão em andamento com o intuito de esclarecer essas questões. Dentre elas, destaca-se o estudo fase II do Grupo de Oncologia do Colégio Americano de Cirurgiões (ACOSOG-Z9000), ${ }^{28}$ que tem como finalidade avaliar sobrevida, taxa de recidiva e toxicidade após tratamento adjuvante com imatinib por um ano em pacientes de alto risco (definidos como tamanho tumoral maior que 10 $\mathrm{cm}$, rotura tumoral, hemorragia intra-peritoneal, tumores intraperitoneais multifocais) submetidos à ressecção completa.

$\mathrm{O}$ estudo fase III, multicêntrico, patrocinado também pelo Colégio Americano de Cirurgiões (ACOSOG-Z9001), ${ }^{28}$ objetiva responder, através da randomização de 380 casos, se existe benefício, a longo prazo, com a utilização do imatinib adjuvante em pacientes portadores de GIST de risco intermediário operados. O grupo de sarcoma ósseo e de partes moles da EORTC ${ }^{28}$ também está avaliando os resultados da terapia adjuvante com imatinib, com projeção de alocação de 400 casos para o ensaio clínico. O ensaio clínico do grupo de sarcoma escandinavo $\left(\right.$ SSGXVII) ${ }^{28}$ espera concluir seu estudo multicêntrico, randomizado, com 80 casos de GIST distribuídos em dois braços (400 mg de imatinib adjuvante por 12 ou 36 meses).

O tratamento neoadjuvante com imatinib também está sendo testado, tendo como exemplo o estudo RTOG-S0132 28 , que visa a avaliar o impacto dessa abordagem na sobrevida livre de progressão e na taxa de resposta objetiva.

A conclusão destes trabalhos revelará se os resultados do tratamento cirúrgico poderão ser otimizados com a associação do imatinib, estabelecendo novas diretrizes no manejo do GIST.

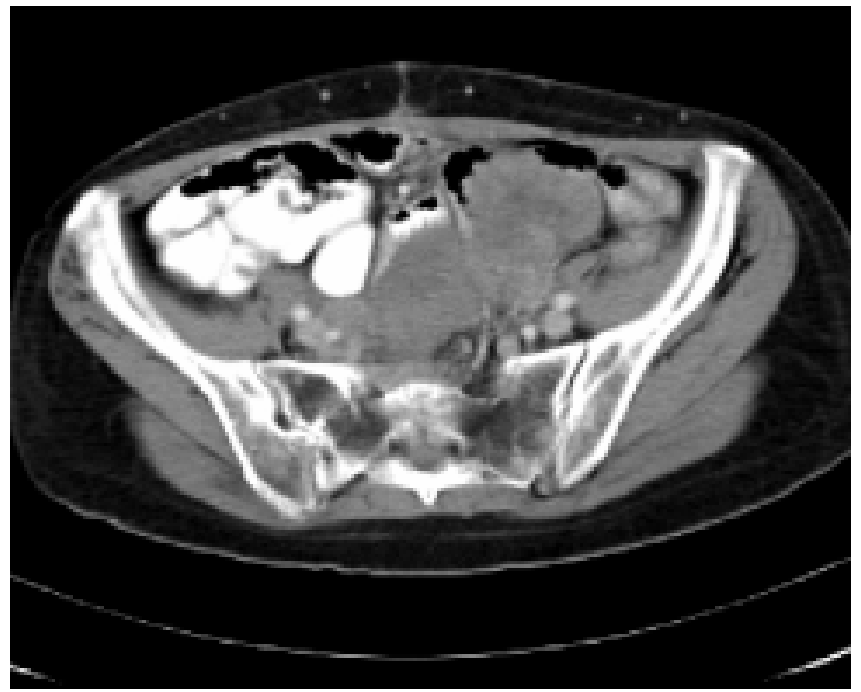

Figura 5 - Após 4 meses de imatinib. 


\begin{abstract}
Gastrointestinal stromal tumor(GIST) has been recently the subject of considerable clinical and experimental interest. This focus is based on insights gained during the past years concerning its identification as a distinct clinical entity and the advances in knowledge about the diagnosis and management. Historically, surgery has been considered the most effective treatment in spite of its limitations. Others therapeutic approaches have been tryng without success, until the introduction of imatinib. This drug provided hopeful results in the treatment of this neoplasia. The idea that imatinib could improve surgical outcome have led to some clinical trials with the hope that this association (imatinib \& surgery) could achieve good results (Rev. Col. Bras. Cir. 2006; 33(1): 51-54).
\end{abstract}

Key words: Gastrointestinal neoplasms; Digestive system surgical procedures; Disease-free survival; Recurrence.

\section{REFERÊNCIAS}

1. Martin JF, Bazin P, Feroldi J, et al. Tumeurs myoides intramurales de l'estomac; consideration microscopiques a propos de 6 cas. Ann Anat Path. 1960;5:484-97.

2. Mazur MT, Clark HB. Gastric stromal tumors: reappraisal of histogenesis. Am J Surg Pathol. 1983; 7(6):507-19.

3. Herrera GA, Pinto de Morales H, Grizzle WE, et al. Malignant small bowel neoplasm of enteric plexus derivation (plexosarcoma). Light and electron microscopic study confirming the origin of the neoplasm. Dig Dis Sci. 1984;29(3):275-84.

4. Lauwers GY, Erlandson RA, Casper ES, et al. Gastrointestinal autonomic nerve tumors. A clinicopathological, immunohistochemical, and ultrastructural study of 12 cases. Am J Surg Pathol. 1993;17(9):887-97.

5. Kindblom LG, Remotti HE, Aldenborg F, et al. Gastrointestinal pacemaker cell tumor (GIPACT): gastrointestinal stromal tumors show phenotypic characteristics of the interstitial cells of Cajal. Am J Pathol. 1998;152(5):1259-69.

6. Hirota S, Isozaki K, Moriyama Y, et al. Gain-of-function mutations of c-Kit in human gastrointestinal stromal tumors. Science. 1998;279(5350):577-80.

7. Isozaki K, Hirota S, Nakama A,et al. Disturbed intestinal movement, bile reflux to the stomach, and deficiency of c-Kit expressing cells in Ws/Ws mutant rats. Gastroenterology. 1995;109(2):456-64.

8. Huizinga JD, Thuneberg L, Kluppel M, et al. W/kit gene required for intestinal cells of Cajal and for interstitial pacemaker activity. Nature. 1995;373(6512):347-9.

9. DeMatteo RP, Lewis JJ, Leung D, et al. Two hundred gastrointestinal stromal tumors: recurrence patterns and prognostic factors for survival. Ann Surg. 2000;231(1):51-8.

10. Heinrich MC, Corless CL, Duensing A, et al. PDGFRA activating mutations in gastrointestinal stromal tumors. Science. 2003; 299(5607):708-710. Epub 2003 Jan 9.

11. Valadão M, Linhares E, Castro L, et al. GIST gástrico. Experiência do INCA. Rev Bras Cancerol. 2004;50(2):121-6.

12. Fujimoto Y, Nakanishi Y, Yoshimura K, et al. Clinicopathologic study of primary malignant gastrointestinal stromal tumor of the stomach, with special reference to prognostic factors: analysis of results in 140 surgically resected patients. Gastric Cancer 2003;6(1):39-48.

13. Mochizuki Y, Kodera Y, Ito S, et al. Treatment and risk factors for recurrence after curative resection of gastrointestinal stromal tumors of the stomach. World J Surg. 2004;28(9):870-5.

14. Wong NA, Young R, Malcomson RD, et al. Prognostic indicators for gastrointestinal stromal tumours: a clinicopathological and immunohistochemical study of 108 resected cases of the stomach. Histopathology. 2003;43(2):118-26.

15. Fletcher CD, Bermen JJ, Corless C, et al. Diagnosis of gastrointestinal stromal tumors: a consensus approach. Hum Pathol. 2002;33(5):459-65.
16. Roberts PJ, Eisenberg B. Clinical presentation of gastrointestinal stromal tumors and treatment of operable disease. Eur J Cancer. 2002;38(suppl 5):537-8.

17. Singer S, Rubin BP, Lux ML, et al. Prognostic value of kit mutation type, mitotic activity, and histologic subtype in gastrointestinal stromal tumors. J Clin Oncol. 2002;20(18):3898-905.

18. Ng EH, Pollock RE, Munsell MF, et al. Prognostic factors influencing survival in gastrointestinal leiomyosarcomas. Implications for surgical management and staging. Ann Surg. 1992;215(1):68-77.

19. Rothlin M, Schob O. Laparoscopic wedge resection for benign gastric tumors. Surg Endosc. 2001; 15(8):893-5. Epub 2001 May 14.

20. Cueto J, Vazquez-Frias JA, Castaneda-Leeder P, et al. Laparoscopic-assisted resection of a bleeding gastrointestinal stromal tumor. JSLS. 1999;3(3):225-8.

21. Otani Y, Ohgami M, Igarashi N, et al. Laparoscopic wedge resection of gastric submucosal tumors. Surg Laparosc Endosc Percutan Tech. 2000;10(1):19-23.

22. Mudan SS, Conlon KC, Woodruff JM, et al. Salvage surgery for patients with recurrent gastrointestinal sarcoma: prognostic factors to guide patient selection. Cancer. 2000; 88(1):66-74.

23. Pidhorecky I, Cherney RT, Kraybill WG, et al. Gastrointestinal stromal tumors: current diagnosis, biologic behavior, and management. Ann Surg Oncol. 2000;7(9):705-12.

24. Joensuu H, Roberts PJ, Sarlomo-Rikala M, et al. Effect of tyrosine kinase inhibitor STI571 in a patient with a metastatic gastrointestinal stromal tumor. $\mathrm{N}$ Engl J Med. 2001;344(14):1052-6.

25. van Oosterom AT, Judson I, Verweij J, et al. Safety and efficacy of imatinib (STI571) in metastatic gastrointestinal stromal tumors: a phase I study. Lancet. 2001;358(9291):1421-23.

26. Demetri GD, von Mehren M, Blanke CD, et al. Efficacy and safety of imatinib mesylate in advanced gastrointestinal stromal tumors. N Engl J Med. 2002;347(7): 472-80.

27. Lehnert T. Gastrointestinal sarcoma (GIST) - a review of surgical management. Ann Chir Gynaecol. 1998;87(4):297-305.

28. Eisenberg B, Judson I. Surgery and imatinib in the management of GIST: Emerging approaches to adjuvant and neoadjuvant therapy. Ann Surg Oncol 2004; 11: 465-475.

29. Martin JF, Bazin P, Feroldi J, et al. Tumeurs myoides intramurales de l'estomac; consideration microscopiques a propos de 6 cas. Ann Anat Path. 1960;5:484-97.

Endereço para correspondência:

Eduardo Linhares

Av. Visconde de Albuquerque, 1102/201

Leblon

22450-000 - Rio de Janeiro - RJ

E-mail: eduardol@inca.gov.br 